\title{
18
}

\section{An Installed Accuracy Assessment using Dye Dilution Testing for Seven Common Flow Metering Technologies}

Marc C. Stonehouse, Mark J. TenBroek, Gary E. Fujita and Timothy J. Dekker

An accurate dye dilution testing protocol using Rhodamine WT was developed and used to quantify flow meter accuracy in the Greater Detroit Regional Sewer System. Over 150 tests were performed on 37 flow meters in conjunction with a set of good metering practice principles. A summary of the accuracy for each of the seven technologies tested before and after good metering practice is given. The seven technologies assessed are electromagnetic induction meters (magmeters); full-conduit, multiple-path, transit-time meters; full-conduit, single-path, transit-time meters; open-channel, multiple-path, transit-time meters; open-channel, ultrasonic meters; flumes; and weirs. Many meters that are typically considered to be accurate had errors of more than $30 \%$. Some meters had errors that exceeded $70 \%$.

Overall, the average initial system accuracy for system meters was observed to be $\pm 15.0 \%$ of measurement with an overall bias of $6.1 \%$ (underpredicting flow). After implementing the good metering practice principles, and in particular using dye dilution testing as a diagnostic tool, the average accuracy potential observed for the system reduced to $\pm 5.5 \%$ of measurement, with an overall bias of $0.6 \%$ (underpredicting flow). It is concluded that (i) there are observable accuracy differences between flow meter technologies, (ii) objective standards like dye dilution testing are critical to good metering, (iii) verifying installed accuracy is important, even for technologies considered to be highly accurate, and (iv) the simplest technology that can be used is often the best.

Stonehouse, M.C., M.J. TenBroek, G. Fujita and T.J. Dekker. 2001. "An Installed Accuracy Assessment using Dye Dilution Testing for Seven Common Flow Metering Technologies." Journal of Water Management Modeling R207-18. doi: 10.14796/JWMM.R207-18.

(C) CHI 2001 www.chijournal.org ISSN: 2292-6062 (Formerly in Models and applications to Urban Water Systems. ISBN: 0-9683681-4-X) 


\subsection{Introduction}

In recent years, flow balances performed on the Greater Detroit Regional Sewer System (GDRSS) by comparing total flows measured at the WWTP with suburban flows have shown an increase in "unaccounted for" flows. The GDRSS Phase IV project was developed to better understand the sources of flow within the GDRSS collection system and those entering the system through suburban customer flow meters. This work included the installation of new flow meters within the collection system to provide more data for detailed system-wide flow balances.

Quality flow meter data was needed for these flow balances. At the start of the project there was a lack of confidence among flow metering professionals relative to the quality of the flow data being collected in the GDRSS collection system. Therefore, one key objective of the Phase IV project was to reduce the uncertainty in flow metering and establish confidence with collected flow data. To accomplish this, a flow metering task force (FMTF), comprising sewer and metering professionals from the Detroit Water and Sewerage Department (DWSD) and the suburban customers, was formed to develop a consensus among system stakeholders on the best flow metering devices, appropriateness of these technologies for different locations, installation methods, and techniques used to evaluate meter accuracy.

One important outcome of the FMTF work was the development of a set of good metering practice guidelines. As part of this effort, an accurate dye dilution testing protocol was developed and used as an objective standard to assess the installed accuracy of over 37 flow meters. The use of good metering practice in conjunction with dye testing as a diagnostic tool has resulted in a significant improvement in system accuracy. Prior to good metering practice, many meters that were considered accurate were found to have errors of more than $30 \%$. This chapter describes the techniques used to quantify initial meter accuracy and the accuracy potential reached under a rigorous operation and maintenance program. Also discussed are some of the typical maintenance issues that were addressed to improve meter accuracy.

While it is appropriate to present the results, this chapter is not an endorsement of any of the technologies assessed. It is important to understand that there is no one technology that is best for all potential meter sites. Every site has different characteristics (such as flow patterns, velocity profiles, up and downstream system constraints, range of flows and depths), which may make using certain technologies impractical or inaccurate. Most of the new meter installations under this project were in very large interceptors that were previously thought to be un-meterable due to the large range of flow and depths 
seen at each site. The most flexible technology was chosen for these sites, but at the cost of greater complexity. Therefore comparisons between technologies must also consider site characteristics. In this chapter, any sites-specific considerations are highlighted in the discussion section for each technology when appropriate.

\section{GDRSS Metering Network.}

The GDRSS collection system serves almost three million people and covers an area of over 800 square miles $\left(1286 \mathrm{~km}^{2}\right)$. About half the flow generated in the system is from suburban customers of the DWSD. Most of this flow enters the GDRSS collection system through eleven existing high-flow billing meters and another three low-flow billing meters. Until this project was developed, the flows inside the City of Detroit were not directly measured until they entered the WWTP. To quantify these flows, an additional eighteen flow meters were installed in conjunction with the eleven existing meters to better understand flows throughout the system.

Seven different flow meter technologies are present in the GDRSS collection system. The different meter technologies, the number tested, the range of meter sizes, and the manufacturers are given in Table 18.1. Meter size is not necessarily the size of the sewer pipe. For most full conduit meters, the meter size is smaller than the pipe size in order to force closed conduit flow. The meter size of a flume is the width of its throat. The meter size of a weir is the width of the weir. The open channel, multiple-path and ultrasonic meter sizes are equal to the pipe size.

Table 18.1 Meter technologies in the GDRSS system.

\begin{tabular}{|c|c|c|c|c|}
\hline Meter Technology & $\begin{array}{l}\text { Number } \\
\text { Tested }\end{array}$ & $\begin{array}{c}\text { Tests } \\
\text { Performed }\end{array}$ & Meter Sizes & $\begin{array}{l}\text { Manufacturer } \\
\text { Represented }\end{array}$ \\
\hline $\begin{array}{l}\text { Electromagnetic Induction } \\
\text { (magmeter) }\end{array}$ & 3 & 17 & $\begin{array}{c}3-5.5 \mathrm{ft} \\
(0.9-1.7 \mathrm{~m})\end{array}$ & $\begin{array}{l}\text { Bailey \& } \\
\text { Fischer/Porter }\end{array}$ \\
\hline $\begin{array}{l}\text { Full-conduit, Multiple-path } \\
\text { (FC MP) }\end{array}$ & 2 & 7 & $\begin{array}{c}4-10 \mathrm{ft} \\
(1.2-3.0 \mathrm{~m})\end{array}$ & Accusonics \\
\hline $\begin{array}{l}\text { Full-conduit, Single-path } \\
\text { (FC SP) }\end{array}$ & 3 & 14 & $\begin{array}{c}4-5.5 \mathrm{ft} \\
(1.2-1.7 \mathrm{~m})\end{array}$ & $\begin{array}{l}\text { Sparling and } \\
\text { Nusonics }\end{array}$ \\
\hline $\begin{array}{l}\text { Open-channel, Multiple-path } \\
\text { (OC MP) }\end{array}$ & 13 & 68 & $\begin{array}{c}6-24 \mathrm{ft} \\
(1.8-7.3 \mathrm{~m})\end{array}$ & Accusonics \\
\hline $\begin{array}{l}\text { Open-channel, Ultrasonic } \\
\text { (OC U) }\end{array}$ & 5 & 11 & $\begin{array}{c}2-15.5 \mathrm{ft} \\
(0.6-4.7 \mathrm{~m})\end{array}$ & $\begin{array}{l}\text { American } \\
\text { Sigma }\end{array}$ \\
\hline Flume & 12 & 34 & $\begin{array}{c}1.5-5 \mathrm{ft} \\
(0.5-1.5 \mathrm{~m})\end{array}$ & N/A \\
\hline Weir & 2 & 6 & $\begin{array}{c}7-8 \mathrm{ft} \\
(2.1-2.4 \mathrm{~m})\end{array}$ & N/A \\
\hline
\end{tabular}




\section{Field Assessment.}

While laboratory testing under highly controlled conditions can be straightforward with the use of weighing tanks, field testing of in situ accuracy is more complicated. Unfortunately, laboratory-measured accuracy says little about the installed accuracy of a specific meter. Each metering technology has its own specifications and operational parameters that need to be fully understood and field-verified to ensure proper operation. A review of the literature reveals little previous work on accuracy of field installed meters. This is due primarily to the lack of a flexible and economical method to apply an objective standard in the field.

In response to the uncertainty associated with flow metering technologies in practice, NSF International of Ann Arbor, Michigan, is currently working with the U.S. EPA National Risk Management Research Laboratory in Edison, New Jersey and the U.S. EPA Office of Research and Development to develop meter verification protocols under the Environmental Technology Verification (ETV) Program. Their goal is to "accelerate development and commercialization of technologies used in the control and abatement of wet weather flows by providing independent third-party verification and reporting of performance" (NSF, 1999). In doing so, they are developing both a laboratory and field protocol for meter verification. The field efforts will include the use of tracer dilution testing as the objective standard for comparison. However, as this chapter will show, even a proven technology must be field-certified at its installed location on a routine basis as many variables affect a meter's performance.

\subsection{Methods}

\section{Good Metering Practice.}

Dye dilution testing is only one element of the good metering practice guidelines developed by the FMTF. These guidelines include procedures for meter evaluation and certification, data recording and review, meter maintenance, and information sharing (Camp Dresser \& McKee, 1998). These practices are not specific to the GDRSS collection system and can be applied to any collection system where flow metering is performed.

The first step of good metering practice is the efficient and timely collection of high-quality meter data. The FMTF recommended that data logging be performed digitally using suitable intervals, typically at a minimum of five minutes, and that logged data include all primary data used by the meter to make estimates of flow. This primary data would include such information 
as the individual sensor velocity and depth measurements, if available. It was also recommended that time averaging over each interval, rather than instantaneous readings, be used to increase the quality of the data. In this project, data was made available remotely via dialup phone lines, and is downloaded on a twice-weekly basis, and data that requires a site visit is downloaded biweekly.

After data is collected, good metering practice requires an ongoing review and assessment of the data. Weekly plots are reviewed at the time of download for meter downtime, flow trends, negative and zero values, depth discrepancies, and other anomalies. A biweekly review is used to compare the data to rainfall records, historical data, gate and dam operations, pump station data, basin data, GIS data, climate data, and recent field calibrations or changes made to the meter. All this data also is reviewed for quality. Meter to meter correlations have been developed and are used to make estimates of missing flows. The dye dilution test results are used first to identify maintenance issues, and secondly to determine adjustment factors, typically ranging from 0.6 to 1.4 , to correct meter data. Redundant-level sensors are used to flag depth sensor drift, and are of great importance in maintaining a high-quality system.

Data review and collection must also be coupled with rapid assessment of equipment problems and clear communication of the tasks required for making field repairs. For this project, weekly meetings between meter data reviewers and field personnel were found to be the most effective way to discuss the issues and assign responsibilities to individuals. Common physical problems identified during the project have included depth sensor drift, failure of ultrasonic transducers, and communications problems. All of the identified problems point to a need for redundant-level measurement and complete logging of all data collected at each meter.

Finally, information sharing is critical for successful operation and maintenance of a system consisting of multiple stakeholders. Information sharing allows all members to understand the operation of the system as a whole, to benefit from maintenance lessons learned throughout the system, and to allow rapid diagnosis and repair of system problems. Components of the shared information should include:

- a description of all meter downtimes and reasons for failure,

- a report of all meter maintenance performed and calibration results,

- a summary of any adjustments made to flow rate calculations,

- a summary of testing performed to verify meter performance, with adjustment factors to indicate discrepancy with a standard testing method, and

- a summary of meter operation: e.g. typical range of flows and levels, total volume metered, and effect of wet weather on flow rate and level. 


\section{Dye Dilution Theory.}

It was determined by the FMTF that certification of system meters should be performed on at least an annual basis, with more frequent and comprehensive testing for meters demonstrating performance problems. The FMTF discussed several options for meter evaluation, including wet well drawdown tests, velocity profiling, temporary metering, and dye dilution testing. Ultimately, the group agreed on the development of a fluorometric dye dilution testing method to be used for meter certification. Dye testing was chosen for its accuracy, flexibility of use for most meter locations, ease of implementation, reliability, and reasonable cost.

The dye dilution method is a means for estimating flow in systems where direct measurement of flow is not possible or is uncertain. A dye dilution test is performed by adding dye to the sewage flow at a constant rate, allowing the dye to mix completely with the sewage flow, and measuring the diluted dye concentration at a downstream location. A mass balance on all fluorescing materials in the system can then be used to estimate the sewage flow, based on the degree of dilution observed at the downstream sampling point (Figure 18.1) as:

$$
\begin{aligned}
& Q_{o} C_{B}+Q_{i} C_{i}=\left(Q_{o}+Q_{i}\right) C_{o} \\
& Q_{o}\left(C_{B}-C_{o}\right)=Q_{i} C_{o}-Q_{i} C_{i} \\
& Q_{o}=\frac{Q_{i} C_{o}-Q_{i} C_{i}}{C_{B}-C_{o}}
\end{aligned}
$$

$\mathrm{C}_{\mathrm{o}}$ can be assumed to be very small relative to $\mathrm{C}_{\mathrm{i}}$, so that:

$$
Q_{o}=\frac{Q_{i} C_{i}}{C_{o}-C_{B}}
$$

where :

$$
\begin{aligned}
Q_{i} & =\text { injected flow rate } \\
C_{i} & =\text { injected concentration, } \\
C_{0} & =\text { concentration in mixed (output) flow, } \\
C_{b} & =\text { background concentration, and } \\
Q_{0} & =\text { output flow rate to be measured. }
\end{aligned}
$$

The analytical technique used in this study eliminates the effect of background fluorescence by using standards prepared from sewage samples. In this case, mass balance on the dye only gives a simplified expression for flow:

$$
Q_{o}=\frac{Q_{i} C_{i}}{C_{o}}
$$


In practice, all measurements of dye concentration are made using standards prepared as dilutions of the injected dye concentration. Thus, fluorescence measurements are used to precisely estimate the degree of dilution obtained in the system, rather than real values of rhodamine concentration. The dye dilution equation can be further simplified to:

$$
Q_{o}=Q_{i} D
$$

where $D$ is the measured dilution of dye, defined as $D=C_{i} / C_{o}$.

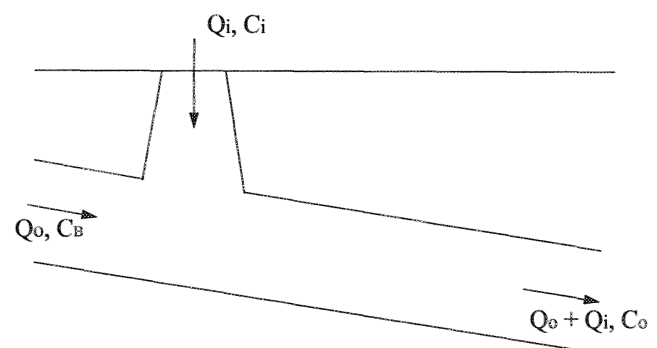

Figure 18.1. Mass balance on all fluorescing materials.

\section{Dye Dilution Protocol.}

While any nonreactive tracer canbe used for estimating flow by this method, the use of fluorescent dye is particularly convenient because simple and accurate fluorometric methods are available for on-site continuous analysis of concentrations. A commonly used fluorometric dye is Rhodamine WT, a dark red dye that is extremely nonreactive and has a very low tendency to adsorb to solid surfaces (Shiau et al., 1993; Smart et al., 1977). Analytical methods for determining Rhodamine WT concentrations are well developed, and concentrations as low as 0.1 parts per billion (ppb) are detectable using standard techniques. Rhodamine dye is invisible to the naked eye at concentrations below approximately $100 \mathrm{ppb}$, and is nonhazardous, biodegradable, and UV-photodegradable. Rhodamine WT is commercially available in a number of forms, including powders, solids, and liquids. Rhodamine WT liquid was adopted as the standard test dye used in this study.

The full protocol for dye dilution testing is detailed in a Dye Dilution Test Protocol (Camp Dresser \& McKee, 1996b). In addition, a description of laboratory studies and error analysis work done to support the development of the protocol is a subsequent Meter Uncertainty Analysis (Camp Dresser \& McKee, 1996c). The experience of the Flow Metering Task Force and GDRSS Technical Committee to date, as documented in these memos, has identified 
several key considerations required for accurate, reliable dye testing. These include:

- continuous monitoring of dye injection rate,

- verification of dye consistency,

- accounting for time of travel,

- high-quality standards curve preparation (use of representative samples),

- frequent (time-specific) standards curve preparation,

- continuous monitoring of suspended solids, fluorescence, and

- comprehensive error analysis

If these issues are all addressed satisfactorily, dye testing can be an accurate and flexible method for flow meter accuracy evaluation.

It should also be noted that dye test accuracy can be enhanced by careful operation of the system being metered. In cases where flow can be controlled by gate or pump station operation, flows can be maintained at constant rates and can be targeted to the specific flow range of interest. For all testing sites, a careful examination of possible operational controls should be made.

In practice, dye injection is performed from a van as shown in Figure 18.2a. The dye solution is mixed in a 30-gallon (113 litre) polyethylene barrel. A constant displacement pump that can draw either directly from the 30 -gallon barrel or from a system of calibrated burettes, is used to inject the dye mix into the flow stream. The burettes are used to measure and verify a constant inject rate by performing drawdown tests every 5 minutes. The burettes are filled by a second pump while the injection pump is drawing from the 30 gallon barrel. A three-way valve is used to control this operation.

Dye withdrawal is performed using a trailer fitted with a small lab as shown in Figure 18.2b. A screened submersible pump is used to bring a sample of the flow stream to a filter bucket on the surface. A second pump, with a finer screen, delivers the sample into the trailer and through a Turner Designs10AU fluorometer and a Hach DR/2000 spectrophotometer. The spectrophotometer is used to monitor suspended solids as high levels and rapid changes can interfere with the test. One-minute readings are automatically recorded on a laptop.

\section{Dye Dilution Uncertainty and Review.}

Dye dilution testing, in general, can range in accuracy from $1 \%$ to $30 \%$ depending on the care and instruments used (U.S. Bureau of Reclamation, 1997), indicating the need for a comprehensive error analysis. However, due to its high potential for accuracy, it is included in the American Society of Mechanical Engineers Performance Test Codes (1992). Using the protocol and comprehensive uncertainty analysis developed under this project, average test errors typically range between $2 \%$ and $5 \%$, but can be as low as $1.5 \%$. The 


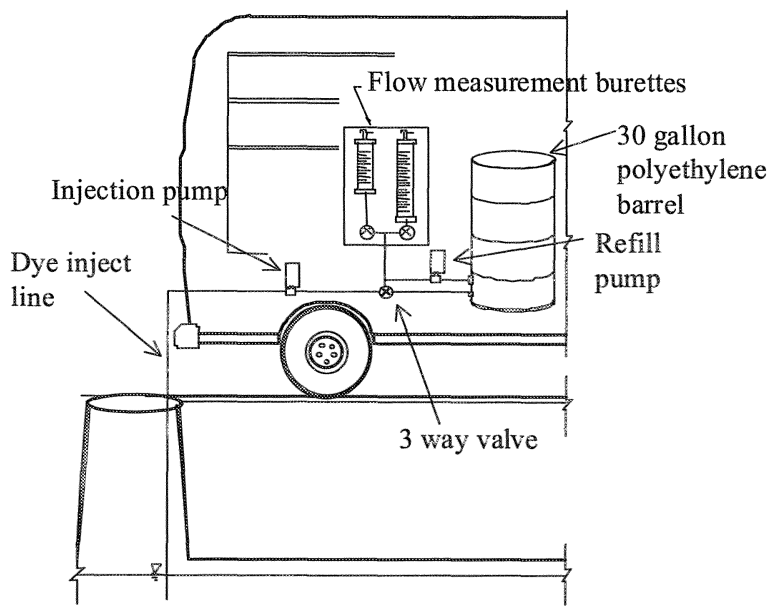

a. Dye inject van.

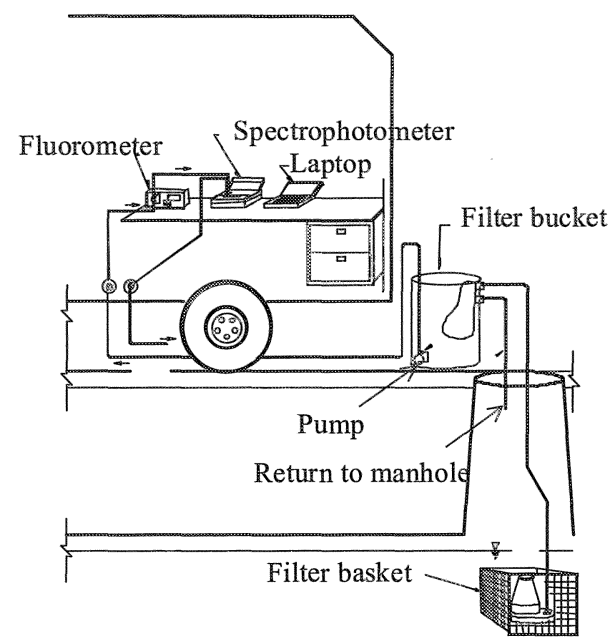

b. Sample collection trailer.

Figure 18.2 Dye inject van and sample collection trailer.

uncertainty of dye test results is calculated at all data points, according to the methods described in Phase IV Technical Memorandum 4 (Camp Dresser \& McKee, 1996c). Dye test uncertainty is a function of:

- uncertainty in injected flow rate,

- uncertainty in measurement of fluorescence,

- error in preparation of standards curves,

- uncertainty in temperature compensation, 
- variation in suspended solids and background fluorescence,

- degree of mixing in the system, and

- uncertainty in adjustment for travel times

The magnitude of each error component is quantified as completely as possible for each flow measurement. In this chapter, total dye test uncertainty is reported as the root sum of squares (RSS) of all error sources, the method commonly used for calculation of $95 \%$ confidence bounds.

Presentations of dye test protocol development, error analysis work, and test verification procedures were made periodically to the FMTF and GDRSS Technical Committee during the development of the protocol in 1996 and early 1997. This included comparisons to a reliable magmeter and wet well drawdown tests. The work was also subjected to review by other contractors, as well as independent reviews by Dr. Steven Wright, professor of hydraulics and hydrology at the University of Michigan. Review of the testing results has been provided by the GDRSS Technical Committee for the duration of the project. The dye testing procedure was accepted by the FMTF as a preferred method for meter evaluation after the initial protocol was developed in 1996, and was formally adopted into the FMTF Guidelines for Good Metering Practice in January 1999.

Unique aspects of the dye testing protocol and example results were also presented at the national conference of the Water Environment Federation (WEF) (Dekker et al., 1997), and at the WEF Special Conference on Advances in Urban Wet Weather Pollution Reduction (Dekker et al., 1998). Discussions are currently underway with the EPA/National Sanitation Foundation to incorporate the dye testing technique into a standard method for meter evaluation.

\section{Meter Accuracy and Bias.}

Metering errors result from numerous error sources that differ between technologies. For example, errors associated with open-channel, transit-time meters could include depth-sensing error, inaccurate location of transducers, inaccurate characterization of the pipe geometry, or approximations made in the integration algorithm. While it is important to understand these error components, a detailed study of the individual sources of accuracy is not attempted in this work. Instead, only the overall error of measurement for a completely installed metering system is found by comparing dye test and meter flows.

Dye test/meter comparisons are made at time points where the total uncertainty in the dye test is minimized, usually in the vicinity of a (time lag compensated) standards curve as shown in Figure 18.3. The dye test results are plotted with $95 \%$ confidence intervals. Each vertical line represents a standards curve. Discrepancy between dye test and meter flows is represented 
by an adjustment factor defined as:

$$
\text { Adjustment Factor }=\frac{\text { Flow }(\text { dyetest })}{\text { Flow }(\text { meter })}
$$

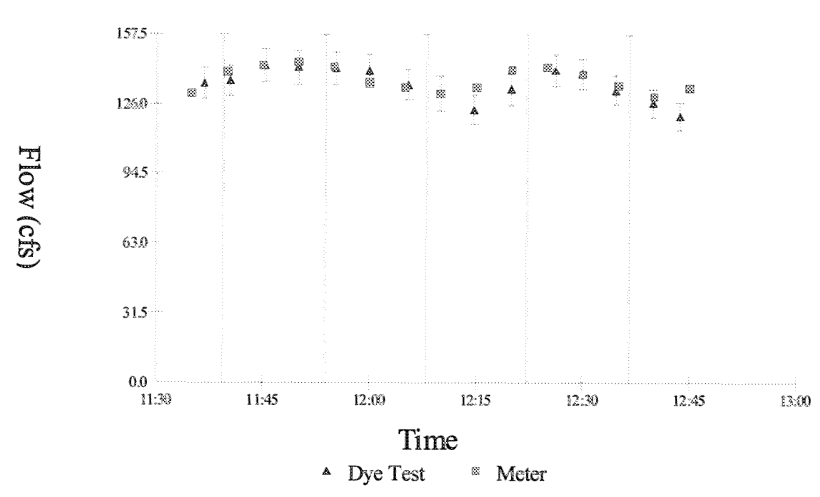

Figure 18.3 Dye test and meter flows compared.

In order to make the most appropriate comparison between dye test and meter data, dye test results are averaged over the same interval used by the data acquisition systems in place at the sites investigated. For all current tests, dye test data averaging follows the convention used by the datalogging equipment of averaging over a 5-minute interval and time-stamping the data with the start time of the interval.

The adjustment factors (AF) calculated at single comparison points during a test are used to find an average test $\mathrm{AF}$ and $95 \%$ confidence interval (average RSS) as shown in Table 18.2. Single-point AFs are also used to estimate the average accuracy of a meter during a period of consistent meter operation, or

Table 18.2 Calculation of the average test adjustment factor.

\begin{tabular}{|c|c|c|c|c|}
\hline \multirow[b]{2}{*}{ Time } & \multicolumn{2}{|c|}{ Flow (cfs) } & \multirow[b]{2}{*}{ Adj. Factor } & \multirow{2}{*}{$\begin{array}{c}\text { Uncertainty } \\
\text { (RSS) }\end{array}$} \\
\hline & Dye Test & Meter & & \\
\hline 11.40 & 136.6 & 140.3 & 0.97 & $5.0 \%$ \\
\hline 11.55 & 142.1 & 142.9 & 0.99 & $5.0 \%$ \\
\hline 12.10 & 130.8 & 130.9 & 1.00 & $5.8 \%$ \\
\hline 12.25 & 141.3 & 142.5 & 0.99 & $4.9 \%$ \\
\hline \multirow[t]{2}{*}{12.35} & 132.7 & 134.6 & 0.99 & $4.9 \%$ \\
\hline & & Average $\mathrm{AF}$ & $0.99 \pm .05$ & $5.1 \%$ \\
\hline
\end{tabular}


an adjustment factor (AF) period. Breaks between periods are defined when changes in meter operation occur due to meter maintenance or performance problems that affect meter operation. Using the single point adjustment factors from a single period, the average accuracy for that period is estimated by:

$$
\% \text { Period Accuracy }=\frac{\sum_{n}|1-A F i|}{n} * 100
$$

The period adjustment factors are used to correct meter readings from that period. Likewise, the bias for each AF period can be found as the average of all single-point adjustment factors for that period:

$$
\text { Period Bias }=\frac{\sum_{n} A F i}{n}
$$

Bias estimates greater than 1 indicate that the meter or group of meters is reading low, while biases less than 1 indicate the readings are high. The average accuracy and bias observed for a flow meter technology is found as the average of the AF period accuracy and bias estimates found using Equations 18.8 and 18.9 for that technology.

\subsection{Results and Discussion}

Most combined sewer system meters are not maintained with the level of effort employed under the GDRSS Phase IV program. In particular, a standard measurement method is not typically used as an indicator of accuracy and maintenance needs. Therefore, the state of the GDRSS collection system meters before good metering practice principles were implemented can serve as an example of the potential inaccuracy of similar systems.

One reason for implementing the good metering practice guidelines was to ensure that all meters were being maintained in a consistent and thorough way. Before these guidelines were implemented, existing meters were maintained at different levels of effort. While, flow data for each meter was at a minimum compared to historical data, only some meters underwent a more thorough data review similar to the recommended good metering practice guidelines. Likewise, monthly preventative maintenance checks were performed on some of the meters, whiles others were only visited when data review suggested a problem existed. Even so, meters that were carefully maintained and thought to be accurate were found by dye dilution testing to be highly inaccurate. 
To date, over 150 dye tests have been performed in the GDRSS on seven different flow-metering technologies. Table 18.3 gives the average accuracy and bias of different technologies in the GDRSS before any maintenance issues indicated by good metering practices (in particular, dye testing) were addressed. This includes all tests performed on existing or new meters over the first year dye testing was performed on each meter, or until corrective maintenance was performed on the meter. This also includes tests for a year after any critical meter component was replaced or was brought back online after being inoperable for over six months (or until corrective maintenance was performed). Dye tests were performed on new meters only after the installations were thought to be performing to their capacity and therefore represent how well a typical installation might be expected to behave.

Table 18.3 Flow meter accuracy: initial conditions.

\begin{tabular}{|c|c|c|c|c|c|c|}
\hline Technology & $\begin{array}{l}\text { Expected } \\
\text { Accuracy }\end{array}$ & $\begin{array}{c}\text { Obs. } \\
\text { Accuracy }\end{array}$ & $\begin{array}{l}\text { Obs. } \\
\text { Bias }\end{array}$ & $\begin{array}{l}\text { No. of } \\
\text { Meters }\end{array}$ & $\begin{array}{l}\text { No. of } \\
\text { Tests }\end{array}$ & $\begin{array}{l}\text { No. of } \\
\text { Comparison } \\
\text { Points }\end{array}$ \\
\hline $\begin{array}{l}\text { Electromagnetic } \\
\text { Induction } \\
\text { (magmeter) }\end{array}$ & $2-5 \%$ & $5.2 \%$ & 1.05 & 3 & 10 & 26 \\
\hline $\begin{array}{l}\text { Full-conduit, } \\
\text { Multiple-path } \\
\text { (FC MP) }\end{array}$ & $2-5 \%$ & $5.4 \%$ & 1.0 & 2 & 2 & 6 \\
\hline $\begin{array}{l}\text { Full-conduit, } \\
\text { Single-path } \\
\text { (FC SP) }\end{array}$ & $5-10 \%$ & $7.8 \%$ & 1.06 & 3 & 10 & 30 \\
\hline $\begin{array}{l}\text { Open-channel, } \\
\text { Multiple-path } \\
\text { (OC MP) }\end{array}$ & $5-10 \%$ & $18.6 \%$ & 1.10 & 13 & 43 & 194 \\
\hline $\begin{array}{l}\text { Open-channel, } \\
\text { Ultrasonic } \\
\text { (OC U) }\end{array}$ & $5-20 \%$ & $21.2 \%$ & 1.10 & 6 & 11 & 51 \\
\hline Flume & $5-15 \%$ & $11.8 \%$ & 0.97 & 12 & 25 & 119 \\
\hline Weir & $5-20 \%$ & $27.4 \%$ & 1.27 & 2 & 6 & 29 \\
\hline
\end{tabular}

For comparison, the "expected accuracy" of each meter technology as determined by the FMTF at the beginning of the project is shown in column 2 of Table 18.4. This range of values is derived from an experience-based assessment of the accuracy of each technology. Also given in the final three columns is the total number of meters, tests, and comparison points used to calculate the observed accuracy and bias.

A summary of the observed accuracy for each of the seven technologies after applying good metering practice is given in Table 18.4. In an effort to determine the accuracy potential after using good metering practice, only AF 
periods when no maintenance issues were known to exist were used. This also includes initial test periods if no maintenance issues were identified. The potential realized may not necessarily represent the most accurate conditions under which the meter can operate, but is a measure of the accuracy potential observed at each meter after a greater-than-usual effort has been used to ensure proper operation.

Table 18.4 Flow meter accuracy: observed accuracy potential using good metering practice.

\begin{tabular}{lcccccc}
\hline & $\begin{array}{c}\text { Expected } \\
\text { Accuracy }\end{array}$ & $\begin{array}{c}\text { Obs. } \\
\text { Accuracy }\end{array}$ & $\begin{array}{c}\text { Obs. } \\
\text { Bias }\end{array}$ & $\begin{array}{c}\text { No. of } \\
\text { Meters }\end{array}$ & $\begin{array}{c}\text { No. of } \\
\text { Tests }\end{array}$ & $\begin{array}{c}\text { No. of } \\
\text { Comparison } \\
\text { Points }\end{array}$ \\
\hline $\begin{array}{l}\text { Electromagnetic } \\
\text { Induction } \\
\text { (magmeter) }\end{array}$ & $2-5 \%$ & $4.2 \%$ & 1.04 & 3 & 15 & 52 \\
$\begin{array}{l}\text { Full-conduit, } \\
\begin{array}{l}\text { Multiple-path } \\
\text { (FC MP) }\end{array}\end{array}$ & $2-5 \%$ & $3.8 \%$ & 0.99 & 2 & 4 & 12 \\
$\begin{array}{l}\text { Full-conduit, } \\
\text { Single-path } \\
\text { (FC SP) }\end{array}$ & $5-10 \%$ & $4.5 \%$ & 1.01 & 3 & 12 & 39 \\
$\begin{array}{l}\text { Open-channel, } \\
\begin{array}{l}\text { Multiple-path } \\
\text { (OC MP) }\end{array}\end{array}$ & $5-10 \%$ & $5.8 \%$ & 1.01 & 11 & 40 & 193 \\
$\begin{array}{l}\text { Open-channel, } \\
\text { Ultrasonic } \\
\text { (OC U) }\end{array}$ & $5-20 \%$ & $7.7 \%$ & 0.98 & 4 & 7 & 30 \\
$\begin{array}{l}\text { Flume } \\
\text { Weir }\end{array}$ & $5-15 \%$ & $5.1 \%$ & 1.00 & 5 & 12 & 47 \\
\hline \begin{tabular}{l} 
Weir \\
\hline
\end{tabular} & $5-20 \%$ & - & - & - & - & - \\
\hline
\end{tabular}

\section{Magmeters.}

Electromagnetic induction meters, more commonly called magmeters, operate on the basis of Faraday's principle, which states that flow of a conductor through a magnetic field induces a current proportional to the flow rate. The meters are widely used in water distribution and wastewater collection systems, as they are considered to be accurate and reliable, requiring little maintenance. Their accuracy and consistency stems from directly measuring flow rate without the need to measure depth and velocity.

Magmeters are calibrated in a laboratory prior to installation, but require periodic re-calibration. This may be performed using pump curves or drawdown tests in the field if necessary. Manufacturers claim accuracy as high as $0.5 \%$ of measurement (Camp Dresser \& McKee, 1996a; Mills 1992). The GDRSS FMTF estimated the accuracy to range between 2 and $5 \%$ of measurement. In some cases, corrosion or coating of wetted sensors can cause 
inaccuracies (Mills, 1992). The high cost of magmeters typically limit them to pipes under 5 feet ( 0.6 meters) in diameter, but installations as large as 10 feet (3.0 meters) are possible. In this study, seventeen tests were performed on three magmeters ranging from 3 -5.5 feet (0.9-1.7 meters) in diameter.

A plot of the average test adjustment factors used to determine the values in Tables 18.3 and 18.4 is shown in Figure 18.4. Initial conditions of the three magmeters indicated an average accuracy of $5.2 \%$ with a $5 \%$ bias (flows underpredicted). This was just outside of the expected accuracy of 2-5\%. The accuracy potential observed under three years of good metering practice is $4.2 \%$, with a bias of $4 \%$ (flows underpredicted).

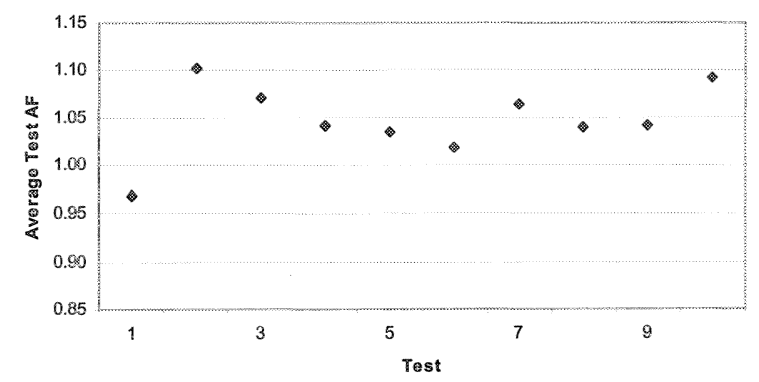

a. Initial conditions.

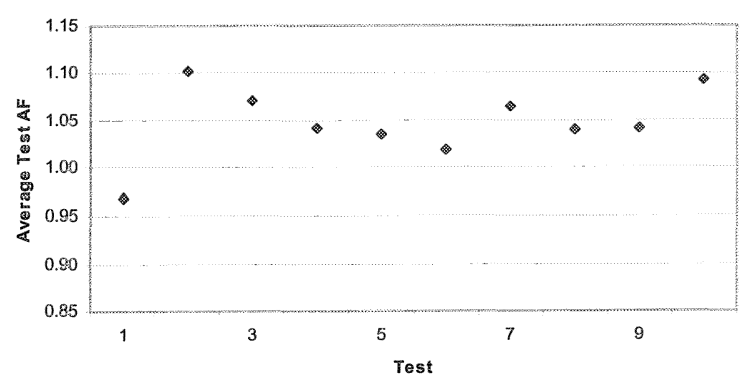

b. Observed potential.

Figure 18.4: Magmeter dye test results.

No corrective maintenance was performed over the three years. The increased accuracy is most likely a statistical anomaly due to the small number of available tests. The results shown in Figure 18.4 reflect the consistent nature 
of the magmeter. However, as shown in Figure 18.5, one of the three magmeters has shown increasing bias over the last 3 years. This issue is currently being investigated, and may be due to sensor corrosion or coating. The final two tests shown in Figure 18.5 are not included in the results of Table 18.4 or Figure 18.4 as the meter is currently under review for a maintenance issue.

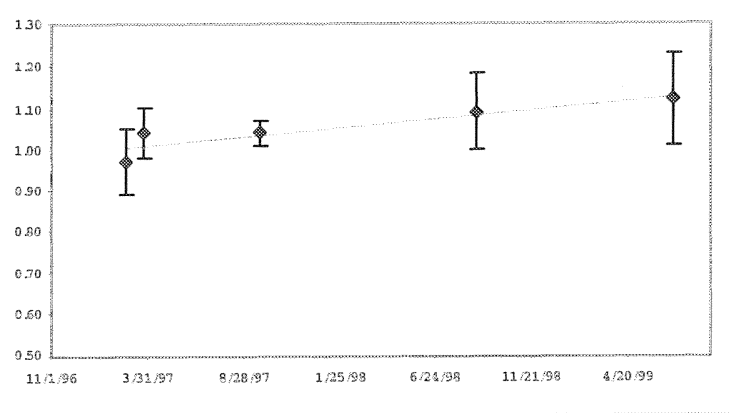

Figure 18.5 Magmeter drift.

The magmeters evaluated showed high accuracy and consistency other than the meter currently under investigation. Other dye tests performed on magmeters at the Detroit wastewater treatment plant not included in this study have indicated a similar bias, underpredicting flows by $2-3 \%$. It is possible that corrosion of the wetted sensors may cause this bias; however, three meters is not a sufficiently large number of samples to allow solid conclusions about a bias in the technology.

\section{FC MP}

Full-conduit, multiple-path (FC MP) meters are area-velocity meters that use two or more acoustic paths to measure the average velocity across each path. They operate under full-pipe conditions so depth sensing is not required. The path velocities are used to determine an average pipe velocity using an integration algorithm. Since the area is well defined and typically 4 or more paths are used, these meters can be highly accurate and consistent if properly maintained. Potential problems include asymmetric velocity profiles, velocity path failures, solids interference, and integration errors (Lowell, 1979 and 1977; Vermeyen 1994; U.S. Bureau of Reclamation, 1997; Patino, 1997). Laboratory and field testing has accuracies ranging from $0.5-2 \%$ in clean water applications (Vermeyen 1994; U.S. Bureau of Reclamation, 1997; Lowell, 1979). Under asymmetric velocity profiles, laboratory testing has found that the integration 
error can be $2-4 \%$ alone (Vermeyen, 1994). Based on experience and engineering judgement, the Flow Metering Task Force estimated an accuracy of $2-5 \%$ in the field for wastewater applications. In this study, four tests were performed on two FC MP meters [4 and 10 feet (1.2 and 3.0 meters) in diameter].

A plot of the average test adjustment factors used to determine the values in Tables 18.3 and 18.4 are shown in Figure 18.6. Initial conditions of the FC MP meters indicated an average accuracy of $5.4 \%$ with no average bias. This was just outside of the expected accuracy of 2.5 and $5 \%$. The accuracy potential observed under three years of good metering practice is $3.8 \%$, overpredicting flows by $1 \%$.

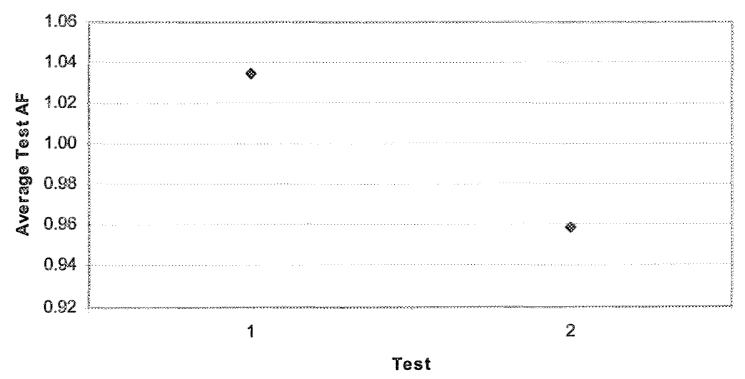

a. Initial conditions.

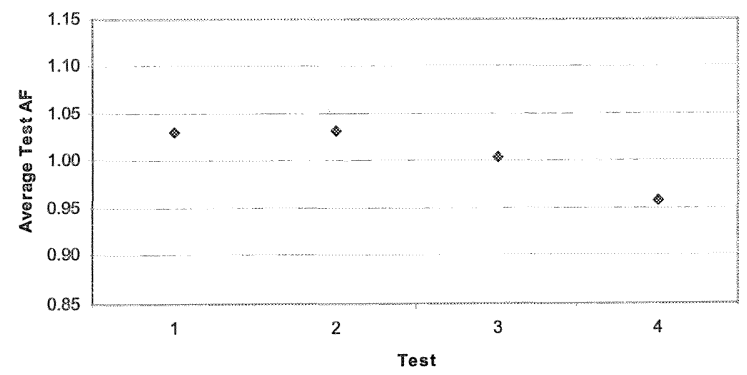

b. Observed potential.

Figure 18.6 FC MP dye test results.

Three of the four tests performed on a 10 -foot-diameter meter indicate a good installation with consistent results (tests 1-3 in Figure 18.6b). However, under certain low flow conditions, it appears that air entrainment can cause path failure. Only one test has been performed on a 4-foot-diameter meter (test 4 
on Figure 18.6b). As review of the base data and dye test results have indicated good operation, no maintenance work has been performed on these meters.

FC SP

Full-conduit single-path meters are similar to FC MP meters except that they use only one path to measure the velocity. The reported accuracy for this technology is as high as $2 \%$ (U.S. Bureau of Reclamation, 1997). The FMTF expected the accuracy of these meters to be between 5-10\%. In this study, fourteen tests were performed on three meters ranging from 4-5.5 feet (1.21.7 meters) in diameter.

A plot of the average test adjustment factors used to determine the values in Tables 18.3 and 18.4 are shown in Figure 18.7. Initial conditions of the FC MP meter indicated an average accuracy of $7.8 \%$ with a bias of underpredicting flows by $6 \%$. This was within the expected accuracy of 5 to $10 \%$. The accuracy potential observed over three years of good metering practice is $4.5 \%$, underpredicting flows by $1 \%$.

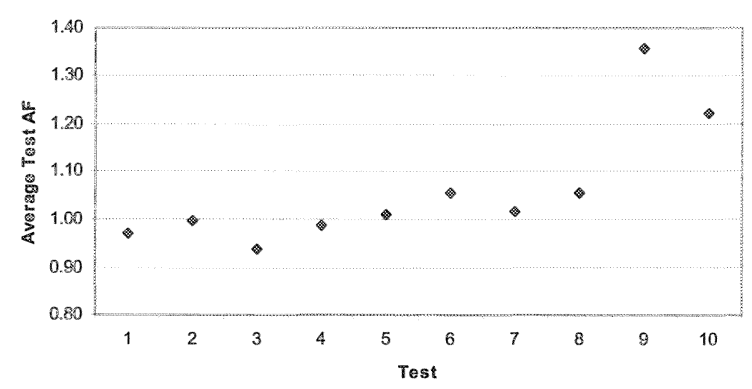

a. Initial conditions.

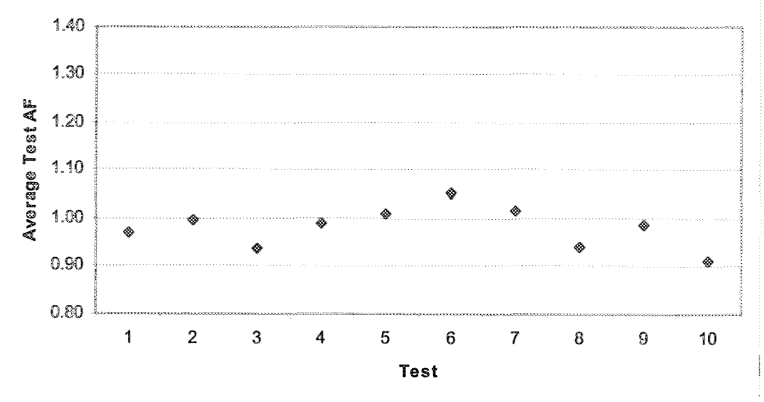

b. Observed potential.

Figure 18.7 FC SP dye test results. 
In general, these three meters have operated accurately and consistently as Figure 18.7 shows. However, one meter, after being off-line for over a year due to a power-line failure, significantly decreased in accuracy. After two dye tests indicated an accuracy of about 30\% (the last two tests plotted in Figure 18.7), an investigation found a faulty transmitter. Dye dilution testing led to a quick identification of the problem and also provided a way to correct the data being collected.

\section{$O C M P$}

Open-channel, multiple-path (OP MP), transit-time meters are similar to the FC MP meters, except they operate under open-channel conditions. The added difficulty of a free surface adds additional error to the flow measurement. A fair amount of laboratory and field-testing has been performed on these meters as they offer the greatest flexibility of use, including rivers and streams. Typically reported accuracy ranges between 3-5\% with at least two paths (Lowell 1979; Lowell 1977). The sources of error are similar to other transit-time meters with the added complexity of integrating velocity in the vicinity of a free surface.

Significant error is also introduced by inaccuracy in depth measurement. All meters tested in this study used two depth sensors of differing technologies (an ultrasonic downlooker and a pressure transducer), to monitor for drift. It was not uncommon at times to find these two measurements to differ by more than 5\%. In most cases the downlooker was used as the primary depth sensor as the pressure transducers had a tendency to drift. In this study, 68 tests were performed on 13 OC MP meters ranging from 6-24 feet (1.8 - 7.3 meters) in diameter.

A plot of the average test adjustment factors used to determine the values in Tables 18.3 and 18.4 are shown in Figure 18.8. Initial conditions of the FC MP meters indicated an average accuracy of $18.6 \%$, with a bias of $10 \%$ (flow underpredicted). This was significantly outside the expected accuracy of 5$10 \%$. The accuracy potential observed over three years of good metering practice is $5.8 \%$, underpredicting flows by $1 \%$.

The OC MP meters are the most complicated metering technology used in the GDRSS system. This complexity is caused by the use of multiple fieldinstalled transducers and a depth sensor that must be accurately installed. In all cases, the meters that were evaluated were new installations where the installation work was performed by a manufacturer's representative using the best available methods to obtain high accuracy. Each meter was installed so that a minimum of two paths would be active under dry weather flow. In some locations, however, the meter had to rely on a single path because of unexpectedly low depths. During single-path operation, the accuracy of these two meters dropped by more than $7 \%$ from double path operation. Dye tests 


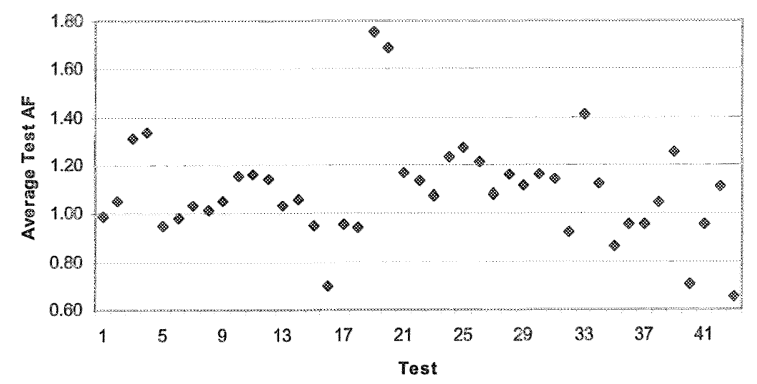

a. Initial conditions.

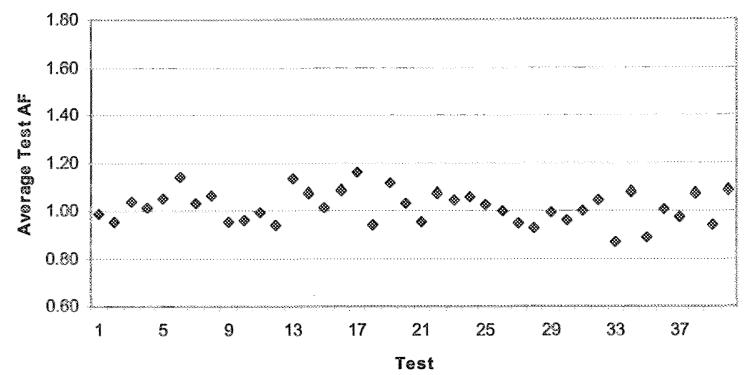

b. Observed potential.

Figure 18.8 OC MP dye test results.

performed under both single- and double-path operation were used to determine site specific algorithms to correct for single-path operation.

It is important to note that most of these meters were installed in locations where no other technology could have been installed. In many cases, these challenging sites were installed without the interruption in flows or during very short and/or incomplete shutdown periods. Perhaps the most challenging aspect of these installations has been providing reliable and accurate depth sensing. As noted above, redundant discrepancies of over $5 \%$ were not uncommon at these locations. This is primarily due to large-pipe diameters that typically have small dry weather water depths with large depth ranges under wet weather conditions.

Because of the complexity of this technology, dye testing was especially useful as it was used to focus the maintenance efforts. Specifically, several meters had errors exceeding 30\% after initial installation, including one meter with an error of over $70 \%$. If dye testing were not used, these meters would 
have been considered to be reasonably accurate. Unfortunately, during the first year of operation for most of these meters, the primary base data, including path velocities and gains, were not recorded. As a result, an important finding of this study was that it is critical to collect and review all the base data for these meters on a weekly basis to monitor for potential issues, such as path failures. It is possible that review of the base data would have indicated some of these errors; however, during each dye test, all base data were collected in the field at the meter in order to confirm proper operation.

Typical corrective actions included re-evaluation of the manufacturer's integration algorithms (which, in some locations, needed to be redeveloped), changes to the pipe geometry, re-evaluation of installed parameters, and adjustment of path heights and lengths. In no cases were the meter parameters "calibrated" to dye test results as a means for increasing accuracy.

Geometry errors due to improperly surveyed parameters can also lead to significant errors. At one location, two meters were installed in cross-plane configurations to each other, each with four paths at the same heights relying on the same depth sensor and data recorder. Measured velocities were found to disagree by more than $10 \%$ on the lower path and $2-4 \%$ on the second and third paths. This has led to an average flow disagreement of over $8 \%$, independent of any depth-sensing error.

The results of this study indicate that these meters can be highly accurate, but an objective standard that enables a certification of installed accuracy is critical for these meters. This is due to the many site-specific variables that affect their accuracy that cannot be found by review of the primary base data.

\section{OC Ultrasonic}

Open-channel (OC), ultrasonic meters, also called Doppler meters, are areavelocity meters that measure the velocity in a flow stream by bouncing acoustic waves off suspended solids or entrained air bubbles. Some technologies use an algorithm to approximate the average velocity based on the maximum velocity observed. Others calculate the average velocity using an analysis of the spectrum of velocities measured. The meters tested in this chapter all used the latter technique to estimate average velocity. A single-depth sensor was used to determine the cross-sectional area of the flow. While these meters can be accurate, testing of various models has shown that they can be inconsistent and have errors as high as $20-30 \%$ of measurement (Trimmer, 1988). The manufacturer of the meters used in this project claim an accuracy of $2 \%$ of measurement (Camp Dresser \& McKee, 1996a). The FMTF estimated an installed accuracy of 5-20\% for this technology. In this study, eleven tests were performed on five OC ultrasonic meters ranging from 2-15.5 feet ( 0.6-4.7 meters) in diameter. 
A plot of the average test adjustment factors used to determine the values in Tables 18.3 and 18.4 are shown in Figure 18.9. Initial conditions of the five OC ultrasonic meters tested indicated an average error of $21.2 \%$ with a bias of $10 \%$ (flows underpredicted). This is outside the expected range of $5-20 \%$. The accuracy potential observed over three years of good metering practice is $7.7 \%$, overpredicting flows by $2 \%$.

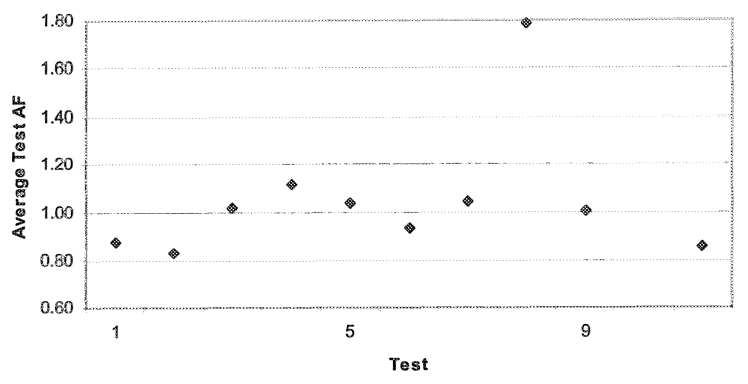

a. Initial conditions.

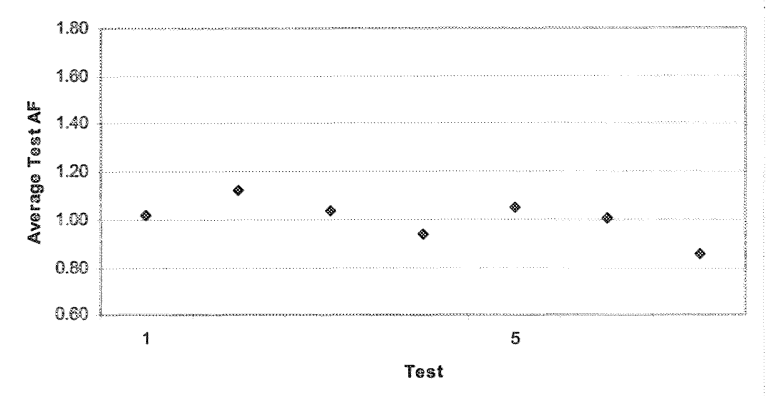

b. Observed potential.

Figure 18.9 OC ultrasonic dye test results.

In general, these meters performed better than expected, having a potential accuracy close to the OC MP meters. As these five meters were temporary installations, operating for less than a year, minimal corrective maintenance was performed. The high initial inaccuracy is primarily due to a single meter that was underpredicting flows by more than $75 \%$. This meter was installed in a challenging location and re-entry to perform corrective maintenance was not possible due to system constraints. Therefore, it is not included in the observed potential assessment. As a result of the dye test evaluations, one meter was upgraded and moved downstream to a more appropriate location. 
Due to the small number of meters and tests performed, no solid conclusions about a technology bias can be made. However, considering that the technology is much simpler to install and maintain compared with OC MP meters, these meters performed very well, with a comparable level of accuracy. It is important to understand that these meters would not be suitable in many of the locations the OC MP meters were installed, as their accuracy will greatly decrease for large diameters pipes with large depths.

\section{Flumes}

Flumes measure flow using an empirically derived depth-discharge relationship. They are very common for wastewater metering as they can be highly accurate and only require depth measurement. The empirical equations have been laboratory verified to $1.5-2.5 \%$ accuracy (U.S. Bureau of Reclamation, 1997) but their field application can have errors of over $10 \%$ (Clemmens, 1988). This can occur from improper construction, distortions caused by settling, and sediment buildup that affect the depth-discharge relationship. Based on experience and engineering judgment, the FMTF estimated an accuracy range of $5-15 \%$ of measurement. In this study, 34 tests were performed on twelve flumes ranging from 1.5-5 feet (0.5-1.5 meters) in width.

A plot of the average test adjustment factors used to determine the values in Tables 18.3 and 18.4 are shown in Figure 18.10. Initial conditions of the 12 flumes tested indicated an average accuracy of $11.8 \%$ with a bias of $3 \%$ (flows overpredicted). This was within the expected accuracy of 5-15\%. After 3 years of good metering practice, the accuracy has increased to $5.1 \%$ with no bias.

The three tests shown in Figure 18.10 with the highest adjustment factors all pertain to a single flume that was affected by a blockage between the stilling basin and the flume. After the third test, the ultrasonic downlooker was relocated over the throat of the flume and the meter is now accurate to within $7 \%$.

Although many of the tests performed around July 1998 are within the GDRSS, they were not under the Phase IV project. These tests were performed only to indicate the meter accuracy, and were not subjected to any further good metering practice maintenance. Therefore, they are only included as initial conditions since no further work was performed to ensure high performance. The observed upward bias seen in the initial conditions is expected with flumes, as typical problems such as sediment buildup or obstructions will cause a restriction of flow, resulting in an increase of depth in the flume measurement section. 


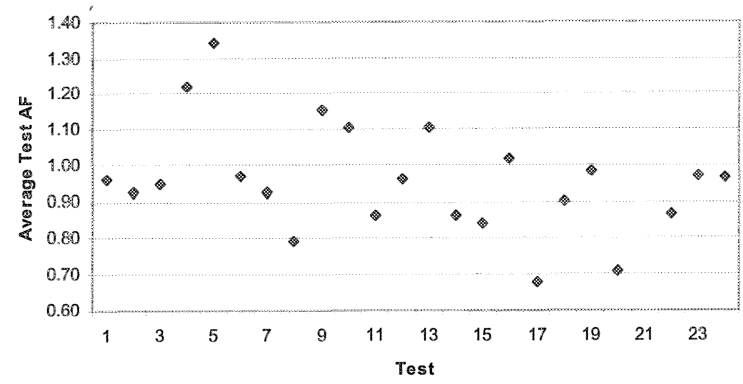

a. Initial conditions.

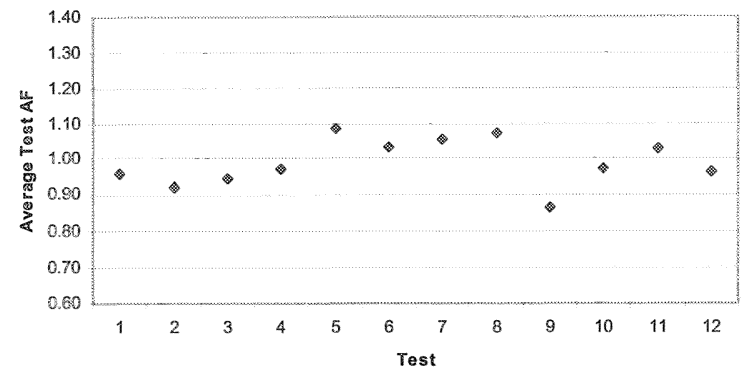

b. Observed potential.

Figure 18.10 Flume dye test results.

Weirs

Weirs also use a known depth-discharge relationship to measure flow. The accuracy of a weir can be as high as 1.5 to $2.5 \%$ (U.S. Bureau of Reclamation, 1997). Weirs suffer the same inaccuracies as flumes, with even greater sedimentation problems as they block flows in the lower portion of the flow stream. The depth of sediment behind a weir can vary from day to day as flows change, making it difficult to apply a consistent depth-discharge relationship. Based on experience and engineering judgment, the FMTF estimated weirs to have an accuracy between 5-20\%. In this study, six tests were performed on two weirs of 7 and 8 feet ( 2.1 and 2.4 meters) in length.

All tests for the weirs are initial conditions as they are both currently under review for maintenance issues, and therefore their observed potential has not yet been seen. A plot of the average test adjustment factors used to determine the values in Tables 18.3 and 18.4 are shown in Figure 18.11. Initial conditions of the two weirs tested indicated an average accuracy of $27.4 \%$ with a bias of $27 \%$ (flows underpredicted). This was significantly outside of the expected accuracy of between 5 and $20 \%$. 


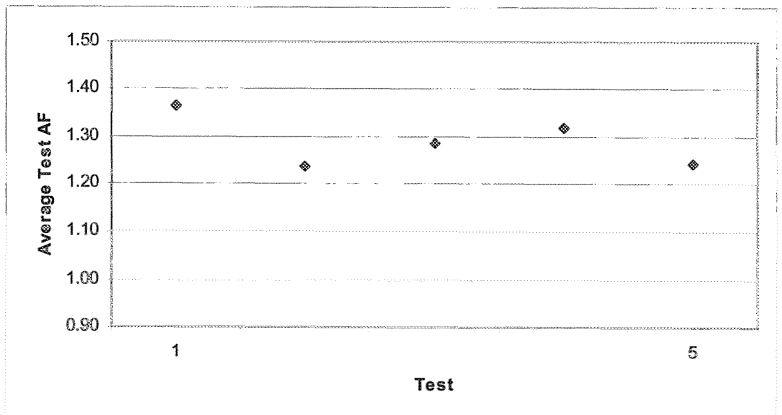

a. Initial conditions.

Figure 18.11 Weir dye test results.

One of the weirs tested, considered accurate at the time and used for billing purposes, was underpredicting flows by $30 \%$. The ultrasonic downlooker was replaced, which increased the accuracy to $23 \%$. More work is still needed to correct these two weirs, which will include a re-evaluation of the weir parameters used for flow calculation. One constant problem with these weirs has been the buildup of sediment behind the weir.

\section{Average System Performance}

Initial conditions of the entire system indicated an average accuracy of $15.0 \%$ with a bias of $6.1 \%$ (flows underpredicted). This was outside the expected accuracy range of 5-10\%. After three years of good metering practice, the observed accuracy potential is $5.5 \%$, underpredicting flows by $0.6 \%$.

Figure 18.12 shows all dye tests used in the accuracy assessment on two plots. As described above, many meters that were or would have been considered reasonably accurate were found to be grossly in error. The observed accuracy potential shown in Figure $18.12 \mathrm{~b}$ still includes some meters that require further investigation, but represents what was accomplished over three years of intensive efforts to bring the system to a satisfactory level of accuracy. More work is needed to continue increasing accuracy.

The overall observed potential bias is extremely low while the initial condition biases are significant enough to adversely affect billing and flow allocation work. The overall initial bias indicates the potential inaccuracies other similar systems may have. A similar bias of underpredicting flows has been seen in water distribution systems, which typically use magmeters (Carney, 1995). However, each wastewater system is variable and complex with its own mix of meter technologies, making it impossible to make a conclusion about bias in other similar systems. This is particularly true as each meter installation is unique, given the site-specific variables such as geometry and hydraulic conditions. 


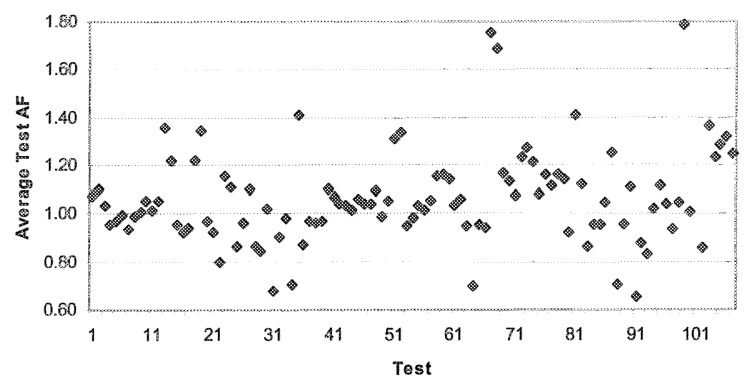

a. Initial conditions.

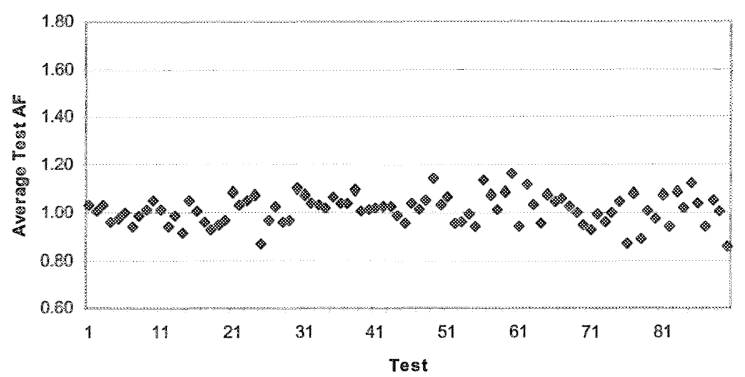

b. Observed potential.

Figure 18.12 All dye test results.

\subsection{Conclusions}

It should be noted that the observed accuracy and bias measurements presented here must be understood in the context of the GDRSS system. Many of the OC MP meter installations were placed in challenging locations previously thought to be un-meterable. Most are in very-large-diameter interceptors [up to 24 feet (7.3 meters) diameter)] flowing only partially full, making depth-sensing accuracy a key component of the overall meter accuracy. Redundant depth sensing at metered locations has periodically shown discrepancies of over $5 \%$. Furthermore, the number of meters tested for most technologies is low $(<10)$ and is not necessarily a representative sample of the entire population. Larger sample sizes would be needed to make any solid conclusions about a particular technology. 
However, several important findings can still be made from the assessment:

1. There are observable accuracy differences for flow meters - The average potential bias for all the meters in the project after applying good metering practice is $0.6 \%$ (underpredicting flows). While the system bias appears to be low, individual metering technologies may have biases or the potential for a significant drift in accuracy. Initial condition testing for flumes, in particular, show a tendency to overpredict flows, as might be expected due to the effects of silt accumulation, physical obstruction, or construction issues. Conversely, the magmeters in the system tended to underpredict flow. However, as expected, magmeters operate consistently and accurately, as evidenced by the observed accuracy of $4.2 \%$. The greatest potential for large inaccuracies was observed for open-channel and complex meters (transit-time and ultrasonic meters). In general, through the use of good metering practice, the different meter technologies all show a good accuracy potential (5-7\%) when rigorously maintained, falling within the expected ranges of accuracy developed by the FMTF. In particular, full-conduit meters show a potential of greater than 5\% accuracy, and should be used when feasible and high accuracy is necessary.

2. Objective standards are important - Having an objective standard such as dye testing was important to the project because it allowed many meter maintenance issues to be identified that may have otherwise remained hidden. In many cases inaccuracies of over $20 \%$ were discovered at meters thought to be accurate. Once corrected, meter accuracy over long periods of time can be tracked with the help of this method. Dye testing has resulted in a considerable improvement in system accuracy, with average error being reduced from $15.0 \%$ to $5.5 \%$.

3. Reviewing installed accuracy is important - As the metering accuracy claimed by manufacturers is developed under controlled laboratory conditions, they typically are not realistic in highly variable and complex field installations. In the process of reviewing meter performance in a variety of field locations, numerous site-specific problems were diagnosed and resolved. For example, the geometry and parameter problems associated with many of the initial Accusonic transit-time installations and the obstruction in one flume's stilling basin would have gone unnoticed. If these reviews are not performed, nothing can be said about the installation accuracy. 
4. Simple is often better - It is important to install the simplest technology possible that meets the accuracy needs. The project staff found that if the flow measurement depends on many different components, errors are often difficult to diagnose and correct. Multi-path transit-time meters are very complex devices that should only be installed if other technologies cannot be costeffectively installed in the locations needed. If complex devices are installed, it is important to have all the data used for calculating flow available so that problems with these data sources can be reviewed and flows re-calculated if necessary.

Based on the metering technologies that were reviewed, primary element systems that use weirs or Parshall flumes can provide a high level of accuracy, if they are properly maintained and if the depth-measurement device is working properly. In addition, magmeters are most applicable to small-pipe installation, and multi-path, transit-time flow meters appear to be the best option, with the level of accuracy needed for large-pipe installations. The FC MP meters compare in accuracy with magmeters and can be installed in large pipes, but have the disadvantage of maintaining a more complex meter. The OC ultrasonic meters compare well OC MP meters for smaller installations with less maintenance required, but would not be applicable for large depths.

The dye dilution testing method developed under this project provides a valuable method for quantifying meter accuracy, which should be used wherever possible to assess meter accuracy. It or another objective standard should be applied consistently to all meters in a collection system if accuracy standards are to be consistently met. The use of dye dilution testing has raised the expectations for average system accuracy. It is now expected that on average the system can reach an accuracy of 5-7\% of measured. Furthermore, technologies such as weirs and flumes should be able to achieve accuracy within $10 \%$.

\section{References}

American Society of Mechanical Engineers, 1992. Performance Test Codes-Hydraulic Turbines and Turbine Mode of Pump/Turbines revision, Performance Test Code Committee, No. 18, New York, NY.

Camp, Dresser \& McKee, 1996a. Technical Memorandum 1: Flow Metering Technology Assessment. Consultant Services Contract No: CS-1249, Detroit Water and Sewerage Department, Detroit, Mich.

Camp, Dresser \& McKee, 1996b. Technical Memorandum 2: Dye Dilution Testing Protocol. Consultant Services Contract No: CS-1249, Detroit Water and Sewerage Department, Detroit, Mich. 
Camp, Dresser \& McKee, 1996c. Technical Memorandum 4: Meter Uncertainty Analysis. Consultant Services Contract No: CS-1249, Detroit Water and Sewerage Department, Detroit, Mich.

Camp, Dresser \& McKee, 1998. Technical Memorandum 8: Guidelines for Good Metering Practice. Consultant Services Contract No: CS-1249, Detroit Water and Sewerage Department, Detroit, Mich.

Carney, Mark C., John Travis, Jeffrey A. Smith, 1995. Better Large Meter Accuracy Increases Revenues, Reduces Water Losses, Water/Engineering and Management, 142(1): 26-28

Clemmens, Albert J., John A. Replogle, Yvonne Reinink, 1988. Visual Estimates of Flume and Weir Operating Conditions, Pro. Of Planning Now for Irrigation and Drainage in the 21st Century. ASCE, New York, NY p651-658.

Dekker, Timothy J., Mark J. TenBroek, Rashila Karsan, Dye Dilution Testing in the Greater Detroit Regional Sewer System- Laboratory Investigation and Field Screening Techniques, Water Environment Federation Wet Weather Specialty Conference, Cleveland, OH, June 1998.

Dekker, Timothy J., Rashila Karsan, Mark J. TenBroek, Steve Wright, Michael Felczak, Ranu Meah, and Ken Prybys, The Use of Dye Dilution Testing for Flow Meters in the Greater Detroit Regional Sewer System, Water Environmental Federation National Conference, Chicago, IL, October 1997.

Lowell, Francis C. JR., Fritz Hirschfeld, 1979. Acoustic Flowmeters for Pipelines, Mechanical Engineering 101(10): 29-35.

Lowell, Francis C. Jr., 1977. Designing Open-channel Acoustic Flowmeters for Accuracy, Part 1, Water and Sewage Works, July and August.

Mills, Raymond C. JR.; 1992 Introduction to Magnetic Flowmeter; Pub. 10D-14a; Bailey-Fisher \& Porter Company, Warminster, Pennsylvania.

NSF International, 1999. Generic Field Protocol for the Verification of Flowmeters for Stormwater and CSO Applications Draft 2.1 Wet Weather Flow Technologies ETV, October 4, 1999.

Patino, Eduardo, Darwin Ockerman, 1997. Computation of Mean Velocity in Open Channels Using Acoustic Velocity Meters, USGS open-file Report 97-220, Tallahassee, Florida

Shiau, B-J., Sabatini, D.A., and Harwell, J.H., 1993. Influence of Rhodamine WT Properties on Sorption and Transport in Subsurface Media. Ground Water 31(6): 913-920.

Smart, P.L., and Laidlaw, I.M.S., 1977. An Evaluation of Some Fluorescent Dyes for Water Tracing. Water Resources Research 13(1): 15-33.

Trimmer, Walter L., Anothony Taylor, Herschel West, 1988. Ultrasonic Flow Meter Testing, Pro. Of Planning Now for Irrigation and Drainage in the 21 st Century. ASCE, New York, NY p643-650.

U.S. Department of the Interior, Bureau of Reclamation, 1997. Water Measurement Manual 3rd ed. The Bureau, Denver, Co.

Vermeyen, Tracy, 1994. Laboratory and Field Evaluation of Acoustic Velocity Meters. Proc. of the Symposium on Fundamentals and Advances in Hydraulic Measurements and Experimentation. ASCE, New York, NY p43-52 
